\title{
Leukocyte Adhesion Deficiency-III: When Leukocytes Cannot Stop
}

$\mathrm{R}$ ecruitment of leukocytes from the vasculature to sites of inflammation involves a highly regulated interplay between adhesion molecules and their ligands. During the recruitment process, circulating leukocytes are locally captured and begin to roll along the inflamed vessel wall, a process that requires the selectin family of adhesion molecules and their respective ligands. During rolling, leukocytes receive signals from the inflamed vessel wall, which induce a further slowing down and eventual firm arrest (adhesion) of the leukocyte to the vessel wall. Firm leukocyte adhesion depends on leukocyte integrins, a family of transmembrane receptors, specialized for anchoring cells to the surrounding matrix or to other cells. After firm adhesion, leukocytes start to spread and begin to slowly crawl along the vessel wall until they find their optimal location for extravasation into tissue.

Several disorders have been described in which distinct steps along the recruitment cascade are defective. These disorders have been termed leukocyte adhesion deficiencies (LAD) and are generally characterized by recurrent infections and marked leukocytosis. So far, three different LADs (LAD I-III) have been reported. Although the genetic defects underlying LAD-I and -II have been known for several years, the precise molecular basis of LAD-III has only recently been identified. In children with LAD-I, mutations in the $\beta_{2}$ integrin gene, which is specifically expressed in leukocytes, results in a profound defect in leukocyte adhesion giving rise to serious infections in early life and high infant lethality. LAD-II, also known as congenital disorder of glycosylation IIc (CDG IIc), primarily affects leukocyte capture and rolling and is caused by a defective sugar transporter leading to disturbed glycosylation of selectin ligands. Infections in LAD-II are often milder and patients usually survive into adulthood. LAD-III resembles in many aspects the clinical presentation of LAD-I and was initially described as LAD-I/variant, though this name is delusive as the molecular defect underlying LAD-III is distinct from LAD-I. Analogous to LAD-I, children with LAD-III may show delayed separation of the umbilical cord and suffer from frequent serious infections mainly localized to the skin and soft tissues. In contrast to LAD-I, patients with LAD-III demonstrate a lifethreatening Glanzmann-like bleeding tendency caused by defective platelet aggregation. Molecular characterization of leukocytes isolated from LAD-III patients revealed that $\beta_{1}, \beta_{2}$, and $\beta_{3}$ integrins are normally expressed. However, they remain inactive even under situations that usually activate them. This observation suggested that a mutation in an integrin regulatory protein, specifically expressed in hematopoietic cells, is responsible for the leukocyte and platelet defects.

A first indication for the integrin-binding protein Kindlin-3 as the responsible gene mutated in LAD-III came from studies in Kindlin-3 knockout mice. These mice exhibit a severe bleeding tendency and a leukocyte adhesion defect reminiscent to the phenotype in LAD-III patients. Kindlin-3 belongs to a family of three cytoplasmic proteins, Kindlin-1 to -3. Its expression is restricted to blood cells and it directly binds to intracellular domains of integrins promoting integrin activation together with talin, another integrin-binding protein. Based on these studies several independent laboratories have recently identified mutations in the human Kindlin-3 gene (FERMT3) and corroborated that loss of Kindlin-3 causes LAD-III in man.

At present, bone marrow transplantation is the only curative treatment for patients with LAD-III and has been successfully performed in children with this disorder. Despite the advances in understanding the etiology of LAD-III, many questions remain unanswered. Unraveling the exact molecular mechanism of integrin activation is a challenge of ongoing research and a prerequisite for new therapeutic approaches including gene therapy-Claudia Nussbaum, Markus Moser, Markus Sperandio.

\section{REFERENCES}

1. Moser M, Bauer M, Schmid S, Ruppert R, Schmidt S, Sixt M, Wang HV, Sperandio M, Fässler R 2009 Kindlin-3 is required for $\beta 2$ integrin-mediated leukocyte adhesion to endothelial cells. Nat Med 15:300-305

2. Svensson L, Howarth K, McDowall A, Patzak I, Evans R, Ussar S, Moser M, Metin A, Fried M, Tomlinson I, Hogg N 2009 Leukocyte adhesion deficiency-III is caused by mutations in KINDLIN3 affecting integrin activation. Nat Med 15:306-312 